\title{
Associação entre manifestações estomatológicas e características socioeconômicas e culturais de crianças brasileiras e norte-americanas infectadas pelo HIV
}

\author{
Liliane J. Grando, ${ }^{1}$ Liliane S. Yurgel, ${ }^{2}$ Denise C. Machado, ${ }^{2}$ Sharon Nachman, ${ }^{3}$ \\ Fred Ferguson, ${ }^{3}$ Barbara Berentsen ${ }^{3}$ e Ângela Fernandes ${ }^{4}$
}

RESUMO Objetivo. Investigar as características socioeconômicas e culturais de crianças infectadas pelo HIV e de suas famílias, relacionando-as com a presença de manifestações estomatológicas da AIDS, e identificar as manifestações estomatológicas mais freqüentes na amostra estudada. Método. A amostra foi composta por 184 crianças de ambos os sexos, com idade entre 0 e 13 anos, atendidas no Hospital de Clínicas de Porto Alegre e Hospital São Lucas da Pontifícia Universidade Católica, em Porto Alegre, Brasil, e no hospital universitário da Universidade do Estado de Nova Iorque em Stony Brook, Estado de Nova Iorque, Estados Unidos. Os dados foram analisados pelos testes do qui-quadrado, $t$ de Student e análise de variância.

Resultados. Dos 184 pacientes, 63,59\% eram do Hospital de Clínicas de Porto Alegre; 22,28\% do hospital de Stony Brook; e 14,13\% do Hospital São Lucas. Na amostra brasileira, $42,66 \%$ das crianças residiam com os pais naturais (principalmente com a mãe), enquanto que nos Estados Unidos $56,10 \%$ das crianças moravam com pais adotivos. Em relação à renda, $39,86 \%$ das famílias no Brasil ganhavam entre 180 e 450 dólares mensais; 33,57\% não tinham renda ou não forneceram seus dados. Nos Estados Unidos, a pergunta sobre renda não foi autorizada, mas todas as famílias foram classificadas pelos assistentes sociais do hospital como tendo renda abaixo de 1000 dólares. O HIV foi adquirido por transmissão vertical em 97,20\% dos casos no Brasil e $97,56 \%$ nos Estados Unidos. A freqüência das manifestações estomatológicas foi maior nas crianças brasileiras $(72,73 \%)$ do que nas norte-americanas $(53,66 \%)(\mathrm{P}=0,01)$.

Conclusões. As condições socioeconômicas e culturais, estrutura e renda familiar, acesso a informação sobre AIDS e a adesão aos esquemas terapêuticos influenciaram a freqüência das manifestações estomatológicas nas duas amostras.

Palavras-chave AIDS, boca, criança, HIV, infecção, medicina bucal.

Universidade Federal de Santa Catarina, Florianópolis (SC), Brasil. Correspondência e pedidos de separatas devem ser enviados a esta autora no seguinte endereço: Rua Dep. Antônio Edu Vieira 147, Bloco A, apto 301, CEP 88040-000, Florianópolis, SC, Brasil. Fone: +55-48-333-6970; e-mail: ljgrando@icablenet.com.br

2 Pontifícia Universidade Católica do Rio Grande do Sul, Porto Alegre (RS), Brasil.

3 State University of New York at Stony Brook, NY, Estados Unidos.

4 Universidade Federal do Paraná, Curitiba (PR), Brasil.
A síndrome da imunodeficiência adquirida (AIDS) é uma doença de origem infecciosa, viral, transmissível através do sangue e secreções humanas contaminadas. A AIDS atinge o sistema imunológico do portador, deixando-o predisposto a infecções oportunistas, neoplasias e problemas neurológicos $(1,2)$. Os primeiros casos de AIDS em adultos $(3,4)$ e crianças $(5,6)$ foram relatados no início dos anos 1980. No Brasil, o primeiro registro de AIDS em indivíduos menores de 13 anos de idade foi feito em 1983 (7).

Sabe-se que a transmissão do HIV pode ocorrer através da via sexual, do uso de drogas injetáveis, da transfusão 
de sangue ou derivados contaminados, de mãe para filho (intra-útero, perinatal ou através do aleitamento materno) e através do contato profissional com sangue contaminado. Dentre essas, a via sexual é a principal responsável pela transmissão do HIV em adultos e a transmissão vertical é a principal responsável pela transmissão do vírus para crianças (8).

Em muitas famílias, o diagnóstico da infecção pelo HIV ainda é realizado a partir do adoecimento de uma criança (9-11). Entretanto, o aumento da longevidade e a melhoria da qualidade de vida das crianças infectadas pelo HIV depende, fundamentalmente, do diagnóstico precoce e do adequado tratamento da infecção. É cada vez maior o número de crianças infectadas pelo HIV que sobrevivem, chegando à adolescência ou mesmo à idade adulta (1).

Além de causar anormalidades funcionais significativas no sistema imunológico, a AIDS afeta toda a estrutura econômica e psicoemocional familiar (12). Muitas crianças e adolescentes vivem com pais biológicos portadores da infecção ou doentes. Outras vivem com outros familiares em função do falecimento dos pais. Muitas vivem em instituições por não terem familiares em condições de lhes oferecer assistência; algumas são adotadas (13). Em alguns lugares, essas crianças crescem sem assistência médica, familiar ou institucional adequada (12).

Fatores socioeconômicos e culturais como pobreza, promiscuidade, uso de drogas, criminalidade e perda dos pais ou outros membros da família em função da AIDS representam a realidade de muitos dos adolescentes e crianças infectados pelo HIV (14). Quando um dos pais fica doente, a renda familiar cai e grande parte dos recursos financeiros passa a ser consumida em medicamentos e no tratamento. Assim, as crianças enfrentam a perda gradativa dos pais, com dificuldades econômicas e incertezas crescentes em relação ao futuro. Elas ainda enfrentam discriminação, devido à associação feita entre sua condição de doentes de AIDS e o comportamento dos pais que levou à aquisição do HIV (10).

Assim como outras profissões da área da saúde, a odontologia sofreu e continua a sofrer as conseqüências da AIDS. O grande número de manifestações estomatológicas relatadas na literatura alerta para a importância da realização rotineira de exames clínicos criteriosos da cavidade bucal e da definição de condutas com relação ao tratamento e da promoção da saúde bucal. A importância das manifestações estomatológicas nas crianças infectadas por HIV levou à inclusão dessas manifestações nos critérios de classificação da AIDS pediátrica proposta pelos Centros para a Prevenção e o Controle de Doenças (CDC) em 1994. As manifestações estomatológicas têm sido apontadas como de grande valor prognóstico na avaliação da progressão da doença (15).

Assim, o objetivo do presente estudo foi investigar as características socioeconômicas e culturais de crianças infectadas pelo HIV e de suas famílias, relacionando-as com a presença ou não de manifestações estomatológicas da AIDS, além de identificar as manifestações estomatológicas mais freqüentes na amostra estudada.

\section{MATERIAIS E MÉTODOS}

Este trabalho foi aprovado pelos Comitês de Ética e de Pesquisa dos hospitais envolvidos. O estudo foi realizado no período de junho de 1999 a junho de 2000. Foram incluídas crianças brasileiras e norte-americanas infectadas pelo HIV com idade de 0 a 13 anos $(1,16,17)$, cujas famílias voluntariamente autorizaram a participação das crianças no trabalho. A amostra brasileira foi composta por 143 crianças da Cidade de Porto Alegre, Sul do Brasil. Vinte e seis eram atendidas no ambulatório de AIDS pediátrica do Hospital São Lucas da Pontifícia Universidade Católica do Rio Grande do Sul (PUCRS) e 117 no Hospital de Clínicas de Porto Alegre (HCPA). A amostra norte-americana foi composta por 41 crianças atendidas nos ambulatórios da unidade pediátrica de doenças infecciosas do hospital universitário da Universidade do Estado de Nova Iorque (SUNY), em Stony Brook, Estado de Nova Iorque, Estados Unidos, e da faculdade de odontologia da mesma universidade.

Os pais ou responsáveis pelas crianças incluídas neste trabalho foram orientados a respeito do mesmo e assinaram um termo de consentimento informado. Após o consentimento, foi realizada uma entrevista individual com os familiares ou acompanhantes de cada paciente, com preenchimento de uma ficha clínica contendo dados de identificação do paciente, dados socioeconômicos e o histórico da aquisição do HIV pela criança e pelo pai e mãe naturais. Para o presente estudo, apenas os dados relativos ao histórico materno de aquisição foram considerados.

O exame clínico estomatológico foi realizado com o auxílio de luz artificial, espátula de madeira, gaze, espelho clínico odontológico e afastadores de acrílico para auxiliar uma eventual documentação fotográfica. Todo o instrumental não descartável utilizado foi adequadamente desinfetado, lavado, empacotado em envelopes próprios para esterilização e autoclavado antes da utilização. O material contaminado foi transportado do local de realização dos exames até os centros de esterilização em uma caixa plástica de paredes rígidas, com tampa e boa vedação. $\mathrm{O}$ pesquisador utilizou barreiras de proteção adequadas.

Primeiramente foi realizado o exame extra-bucal da região cérvico-facial (palpação dos linfonodos cérvico-faciais com a criança sentada, de frente para o profissional, com a cabeça ligeiramente inclinada para a frente) e o exame das parótidas (criança deitada na maca ou na cadeira odontológica, com a cabeça ligeiramente inclinada para trás). No exame intra-oral, a criança permaneceu deitada e foi examinada de acordo com a seqüência: região peri-bucal, semimucosa labial, mucosa labial, região das comissuras, vestíbulo bucal, mucosa jugal, região de fundo de sulco, gengiva, rebordo alveolar, palatos duro 
e mole, pilares amigdalianos, soalho de boca, ventre, dorso e bordas da língua. Além da inspeção visual, a palpação bidigital ou bimanual foi realizada sempre que considerada necessária. Todas as alterações da normalidade foram imediatamente anotadas e desenhadas em local apropriado, na ficha clínica.

O diagnóstico clínico das manifestações estomatológicas consideradas neste estudo foi realizado com base nos critérios da Organização Mundial de Saúde (OMS) (18) e na classificação de RamosGomes et al. (19).

Os dados e resultados obtidos foram tabulados em uma planilha do programa Excel e submetidos à análise estatística pelos testes do qui-quadrado $\left(\chi^{2}\right), t$ de Student e análise de variância.

\section{RESULTADOS}

Um total de 184 crianças participaram do estudo, 63,59\% do HCPA; 22,28\% do hospital da SUNY; e $14,13 \%$ do Hospital São Lucas. A tabela 1 mostra a distribuição dos pacientes quanto ao sexo. A semelhança quanto ao número de meninas e meninos foi puramente casual, pois o gênero não foi considerado como critério de seleção da amostra.

A distribuição da amostra de acordo com os hospitais e a faixa etária das crianças pode ser observada na figura 1. Os resultados obtidos com relação ao dado "com quem vive a criança?" são apresentados na figura 2. A maioria das crianças brasileiras que viviam com "pelo menos um dos pais naturais" residiam com a mãe pelo fato de o pai ter abandonado a família tão logo o diagnóstico de AIDS foi realizado nele próprio, na esposa ou na criança. Entre os "outros familiares" destacaram-se os avós, tios ou padrinhos. Tais resultados revelam um grave comprometimento $\mathrm{da}$ unidade familiar em função de doença.

A tabela 2 mostra os dados sobre renda familiar em salários mínimos (um salário mínimo equivalia a
TABELA 1. Distribuição das crianças portadoras de HIV quanto ao sexo, Brasil e Estados Unidos, 1999 e 2000

\begin{tabular}{|c|c|c|c|c|c|c|}
\hline \multirow[b]{2}{*}{ Sexo } & \multicolumn{2}{|c|}{ Brasil ${ }^{a}$} & \multicolumn{2}{|c|}{ Estados Unidos ${ }^{b}$} & \multicolumn{2}{|c|}{$\begin{array}{c}\text { Total } \\
\end{array}$} \\
\hline & No. & $\%$ & No. & $\%$ & No. & $\%$ \\
\hline Feminino & 68 & 47,55 & 19 & 46,34 & 87 & 47,28 \\
\hline Masculino & 75 & 52,45 & 22 & 53,66 & 97 & 52,72 \\
\hline Total & 143 & 100,00 & 41 & 100,00 & 184 & 100,00 \\
\hline
\end{tabular}

a Brasil: 117 crianças do Hospital de Clínicas de Porto Alegre e 26 crianças do Hospital São Lucas da Pontifícia Universidade Católica do Rio Grande do Sul.

${ }^{b}$ Estados Unidos: 41 crianças do hospital universitário e faculdade de odontologia da Universidade do Estado de Nova lorque em Stony Brook.

FIGURA 1. Distribuição das crianças portadoras de HIV segundo hospital e faixa etária, Brasil e Estados Unidos, 1999 e 2000a

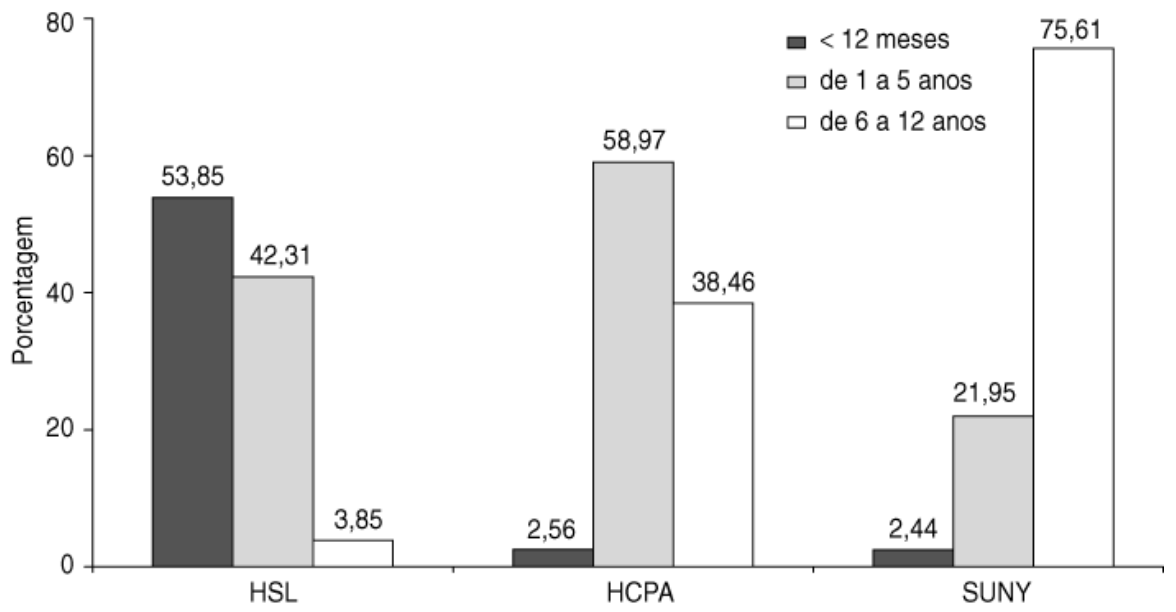

a HSL = Hospital São Lucas da Pontifícia Universidade Católica do Rio Grande do Sul, Brasil; HCPA = Hospital de Clínicas de Porto Alegre, Brasil; SUNY = Hospital universitário da Universidade do Estado de Nova lorque em Stony Brook, Estados Unidos.

FIGURA 2. Distribuição das crianças portadoras de HIV quanto ao responsável, Brasil e Estados Unidos, 1999 e $2000^{a}$

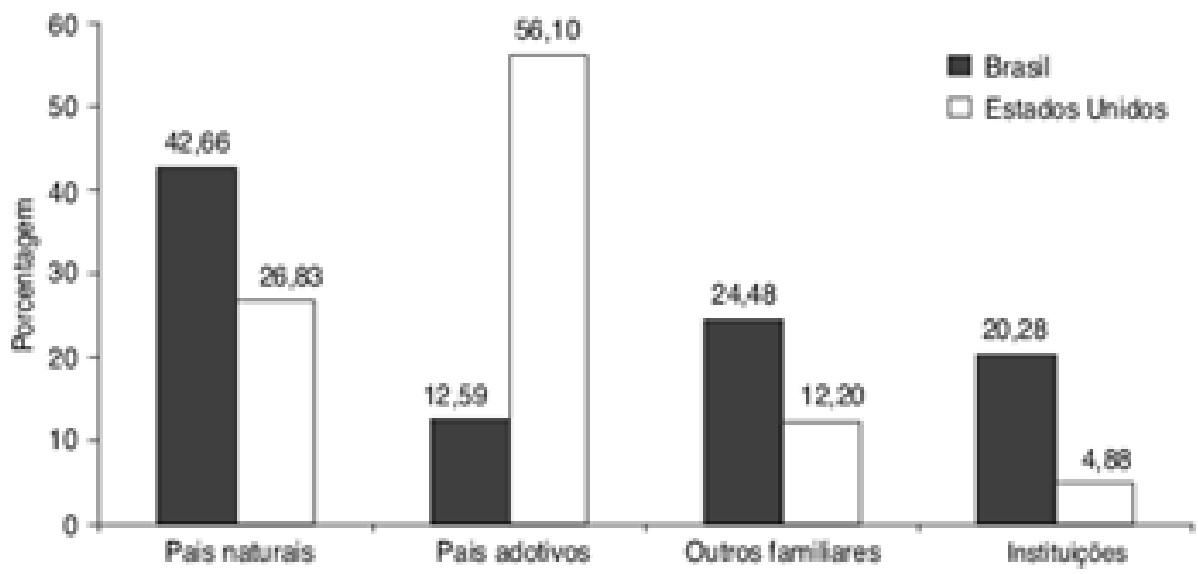

a Brasil: 117 crianças do Hospital de Clínicas de Porto Alegre e 26 crianças do Hospital São Lucas da Pontifícia Universidade Católica do Rio Grande do Sul. Estados Unidos: 41 crianças do hospital universitário e da faculdade de odontologia da Universidade do Estado de Nova lorque em Stony Brook. 
TABELA 2. Distribuição das crianças portadoras de HIV segundo renda familiar, Brasil, ${ }^{a} 1999$ e 2000

\begin{tabular}{lcc}
\hline & \multicolumn{2}{c}{ Distribuição } \\
\cline { 2 - 3 } Renda familiar (salários mínimos) & No. & $\%$ \\
\hline Um & 20 & 13,99 \\
De dois a cinco & 57 & 39,86 \\
Acima de seis & 18 & 12,59 \\
Sem renda ou sem dados & 48 & 33,57 \\
Total & 143 & 100,00 \\
& & \\
a 117 crianças do Hospital de Clínicas de Porto Alegre e 26 crianças do Hospital São Lucas da \\
Pontifícia Universidade Católica do Rio Grande do Sul. \\
b O valor do salário mínimo no Brasil era de aproximadamente US\$ 90,00 na época do estudo.
\end{tabular}

TABELA 3. Modo de aquisição do HIV pela criança e pela mãe natural, Brasil e Estados Unidos, 1999 e 2000

\begin{tabular}{|c|c|c|c|c|c|c|}
\hline \multirow[b]{2}{*}{ Modo de transmissão } & \multicolumn{2}{|c|}{ Brasila $^{a}$} & \multicolumn{2}{|c|}{ Estados Unidos $^{b}$} & \multicolumn{2}{|c|}{ Total } \\
\hline & No. & $\%$ & No. & $\%$ & No. & $\%$ \\
\hline \multicolumn{7}{|l|}{ Mãe (n = 184) } \\
\hline Sexual & 81 & 56,64 & 13 & 31,71 & 94 & 51,09 \\
\hline Desconhecido & 27 & 18,88 & 11 & 26,83 & 38 & 20,65 \\
\hline Sexual e drogas injetáveis & 15 & 10,49 & 7 & 17,07 & 22 & 11,96 \\
\hline Drogas injetáveis & 10 & 6,99 & 8 & 19,51 & 18 & 9,78 \\
\hline Transfusão de sangue & 4 & 2,80 & 1 & 2,44 & 5 & 2,72 \\
\hline Mãe HIV-negativa & 4 & 2,80 & 1 & 2,44 & 5 & 2,72 \\
\hline Ocupacional & 2 & 1,40 & - & - & 2 & 1,09 \\
\hline \multicolumn{7}{|l|}{ Criança $(n=184)$} \\
\hline Vertical & 139 & 97,20 & 40 & 97,56 & 179 & 97,28 \\
\hline Transfusão de sangue & 3 & 2,10 & - & - & 3 & 1,63 \\
\hline Abuso sexual & 1 & 0,70 & 1 & 2,44 & 2 & 1,09 \\
\hline
\end{tabular}

a Brasil: 117 crianças do Hospital de Clínicas de Porto Alegre e 26 crianças do Hospital São Lucas da Pontifícia Universidade Católica do Rio Grande do Sul.

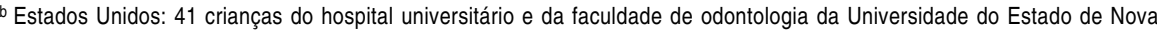
lorque em Stony Brook.

aproximadamente 90 dólares na época do estudo) para a amostra brasileira. Alguns responsáveis pelas crianças não quiseram responder a esta pergunta. $\mathrm{Na}$ amostra norte-americana, não fomos autorizados a questionar sobre renda familiar. Todas as famílias norteamericanas foram classificadas pelos assistentes sociais como sendo de baixa renda (renda mensal inferior a U\$ 1 000,00).

O modo de aquisição do HIV pelas crianças estudadas e por suas mães naturais aparece na tabela 3 . O modo de transmissão sexual foi o mais comum, tanto entre mães brasileiras quanto entre mães norte-americanas.
Das 143 crianças brasileiras, 104 $(72,73 \%)$ apresentaram manifestações estomatológicas. Das 41 crianças norteamericanas, $22(53,66 \%)$ apresentaram essas manifestações. A tabela 4 mostra o tipo de manifestação observada. $\mathrm{O}$ teste do $\chi^{2}$ revelou uma relação significativa entre a presença de manifestações estomatológicas e o país em estudo, com maior freqüência no Brasil $(P=0,01)$.

Linfadenopatia cérvico-facial foi diagnosticada em 173 crianças (94,02\%). Encontraram-se variações quanto ao tamanho, número e posição dos linfonodos. A maioria dos linfonodos palpados encontrava-se assintomático, mole, regular e móvel. A tabela 5 mostra os tipos de terapia antiretroviral em uso pelas crianças da amostra na época em que os exames estomatológicos foram realizados. Durante o período de coleta de dados, não foram observados problemas com relação à adesão das crianças norteamericanas ao tratamento antiretroviral preconizado, embora o contrário tenha sido observado na amostra brasileira. $\mathrm{Na}$ amostra brasileira, houve vários relatos por parte de familiares acerca das dificuldades enfrentadas em entender os esquemas terapêuticos e lembrar dos horários corretos de administração dos medicamentos. O baixo grau de informação e a idade avançada de alguns familiares, especialmente no caso dos avós, também contribuíram para falhas nos esquemas propostos.

\section{DISCUSSÃO}

O ambulatório pediátrico de AIDS do HCPA existe desde 1989, atuando como centro de referência para o atendimento de crianças infectadas por HIV em toda a região metropolitana de Porto Alegre, capital do Estado do Rio Grande do Sul. O ambulatório pediátrico de AIDS do Hospital São Lucas, por sua vez, foi criado em 1997 em função do aumento no número de crianças infectadas pelo HIV nascidas no próprio hospital e em outros hospitais da região. Como o ambulatório do HCPA é mais antigo, as crianças que são tratadas naquele hospital são mais velhas do que aquelas atendidas no Hospital São Lucas.

As unidades pediátricas do setor de doenças infecciosas do hospital universitário e da faculdade de odontologia da SUNY, nos Estados Unidos, se dedicam ao atendimento de crianças infectadas pelo HIV desde 1992. A parceria entre o hospital e a faculdade surgiu da necessidade de um atendimento integrado para o controle e tratamento das doenças estomatológicas e infecções odontogênicas que estariam prejudicando o bom andamento do tratamento médico $(19,20)$. Em função do trabalho em conjunto entre os ambulatórios de pediatria e de gi- 
TABELA 4. Manifestações estomatológicas em crianças portadoras de HIV, Brasil e Estados Unidos, 1999 e 2000

\begin{tabular}{|c|c|c|c|c|c|c|}
\hline \multirow[b]{2}{*}{ Manifestação } & \multicolumn{2}{|c|}{ Brasil $^{\mathrm{a}}$} & \multicolumn{2}{|c|}{ Estados Unidos ${ }^{b}$} & \multicolumn{2}{|c|}{ Total } \\
\hline & No. & $\%$ & No. & $\%$ & No. & $\%$ \\
\hline Aumento de volume das parótidas & 43 & 39,45 & 11 & 40,74 & 54 & 39,71 \\
\hline Candidíase eritematosa & 40 & 36,70 & - & - & 40 & 29,41 \\
\hline Petéquias & 20 & 18,35 & 3 & 11,11 & 23 & 16,91 \\
\hline Gengivite & 14 & 12,84 & 2 & 7,41 & 16 & 11,76 \\
\hline Xerostomia & 8 & 7,34 & 7 & 25,93 & 15 & 11,03 \\
\hline Queilite angular & 11 & 10,09 & 3 & 11,11 & 14 & 10,29 \\
\hline Dermatite seborréica perioral & 7 & 6,42 & 4 & 14,81 & 11 & 8,09 \\
\hline Úlceras bucais & 11 & 10,09 & - & - & 11 & 8,09 \\
\hline Candidíase pseudomembranosa & 9 & 8,26 & 1 & 3,70 & 10 & 7,35 \\
\hline Herpes simples recorrente & 7 & 6,42 & 1 & 3,70 & 8 & 5,88 \\
\hline Eritema gengival linear & 3 & 2,75 & 5 & 18,52 & 8 & 5,88 \\
\hline Molusco contagioso & 5 & 4,59 & - & - & 5 & 3,68 \\
\hline Leucoplasia pilosa & 3 & 2,75 & 1 & 3,70 & 4 & 2,94 \\
\hline $\begin{array}{l}\text { Candidíase hiperplásica } \\
\text { Gengivo-estomatite herpética }\end{array}$ & 4 & 3,67 & - & - & 4 & 2,94 \\
\hline primária (GEHA) & 1 & 0,92 & - & - & 1 & 0,74 \\
\hline Total $^{\mathrm{c}}$ & 186 & - & 38 & - & 224 & - \\
\hline
\end{tabular}

a Brasil: 117 crianças do Hospital de Clínicas de Porto Alegre e 26 crianças do Hospital São Lucas da Pontifícia Universidade Católica do Rio Grande do Sul.

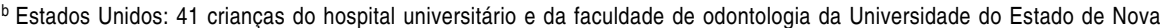
lorque em Stony Brook.

c Algumas crianças apresentaram mais de um tipo de manifestação concomitantemente.

TABELA 5. Tipo de terapia utilizada por crianças portadoras de HIV, Brasil e Estados Unidos, 1999 e 2000

\begin{tabular}{|c|c|c|c|c|c|c|}
\hline \multirow[b]{2}{*}{ Terapia $^{a}$} & \multicolumn{2}{|c|}{ Brasil $^{\mathrm{a}}$} & \multicolumn{2}{|c|}{ Estados Unidos $^{c}$} & \multicolumn{2}{|c|}{ Total } \\
\hline & No. & $\%$ & No. & $\%$ & No. & $\%$ \\
\hline ITRAN & 94 & 65,73 & 15 & 36,59 & 109 & 59,24 \\
\hline ITRAN + ITRANN & 12 & 8,39 & 1 & 2,44 & 13 & 7,07 \\
\hline ITRAN $^{*}$ ou ITRANN ${ }^{b}+$ IP & 19 & 13,29 & 22 & 53,7 & 41 & 22,29 \\
\hline Sem medicação & 18 & 12,58 & 3 & 7,32 & 21 & 11,41 \\
\hline Total & 143 & 100,00 & 41 & 100,00 & 184 & 100,00 \\
\hline
\end{tabular}

a ITRAN = inibidores de transcriptase reversa análogos de nucleosídeo; ITRANN = inibidores de transcriptase reversa nãoanálogos de nucleosídeo; IP = inibidor da protease.

b Brasil: 117 crianças do Hospital de Clínicas de Porto Alegre e 26 crianças do Hospital São Lucas da Pontifícia Universidade Católica do Rio Grande do Sul.

c Estados Unidos: 41 crianças do hospital universitário e da faculdade de odontologia da Universidade do Estado de Nova lorque em Stony Brook.

necologia e obstetrícia, o número de crianças nascidas com a infecção pelo HIV está controlado, fazendo com que a maioria dos pacientes em tratamento encontre-se numa faixa etária mais elevada, na juventude ou na fase adulta.

Da amostra norte-americana estudada, $56,10 \%$ residiam com famílias adotivas. Nos Estados Unidos é grande o número de adoções temporárias ou definitivas de crianças infecção por HIV. Muitas crianças têm adquirido a doença através da transmissão do HIV pelas suas mães. Outras, tais como as crianças de rua, podem adquirir o vírus através do uso de drogas injetáveis ou da promiscuidade infantil. Muitas crianças HIV-negativas também têm vivenciado a realidade da AIDS através do adoecimento de seus pais, familiares ou vizinhos. Os órfãos da AIDS têm se tornado uma realidade; a partir do momento em que um dos pais adquire a infecção por HIV, são grandes as chances de seu parceiro também ser portador do HIV ou desenvolver a doença. Contudo, muitas organizações governamentais e não-governamentais têm se esforçado para atender às necessidades familiares das crianças cujos pais faleceram em decorrência da AIDS; a criação de lares para estas crianças e o estímulo à adoção são bons exemplos disso.

A AIDS afeta toda a estrutura familiar. Após a morte ou abandono dos pais, muitos avós com idade avançada, condições precárias de saúde, situação financeira instável e pouca instrução ou capacidade de entendimento da doença se vêem forçados a cuidar de seus netos (10). Além disso, quando um dos pais fica doente, a renda familiar diminui e os recursos financeiros passam a ser consumidos pelo tratamento médico, podendo levar ao empobrecimento de toda a família (10). Alguns dos responsáveis pelas crianças brasileiras não quiseram responder à pergunta sobre renda. De acordo com o serviço de assistência social do HCPA, a negativa dos familiares pode sugerir a obtenção de recursos financeiros através de meios ilícitos, tais como tráfico de drogas, prostituição e roubo.

$\mathrm{Na}$ amostra norte-americana, todas as famílias foram classificadas pelos assistentes sociais como tendo baixa renda (renda mensal inferior a US\$ $1000,00)$. Apesar disso, observamos que todos os familiares das crianças norte-americanas apresentavam elevado grau de informação a respeito da AIDS comparativamente às famílias brasileiras. O volume de informação recebido pelas famílias nos Estados Unidos através da mídia, 
palestras, reuniões de grupos de apoio e auto-ajuda, cartilhas educativas, fitas de vídeo, livros infantis educativos, propagandas educativas e outros certamente contribuíram para esse aspecto.

As crianças diferem sob muitos aspectos dos adultos em termos de adesão ao tratamento anti-retroviral (16). Geralmente, as crianças infectadas pelo HIV vivem com suas mães também infectadas, dependendo delas para receber os medicamentos. Os esquemas terapêuticos cada vez mais complexos exigem inúmeros cuidados, tais como horário, dosagens, combinações com alimentos, etc. Níveis baixos de compreensão e instrução dos pais ou familiares podem comprometer a adesão da criança ao tratamento, induzindo resistência aos medicamentos e levando a uma piora das condições gerais de saúde (21).

Desde o início da história da AIDS, o perfil epidemiológico da doença mudou muito. Hoje sabe-se que as mulheres são mais suscetíveis à contaminação pela via sexual do que os homens, especialmente se apresentarem úlceras genitais, sangramentos vaginais ou doenças sexualmente transmissíveis, se realizarem sexo anal, se tiverem múltiplos parceiros contaminados ou se o parceiro estiver em fase avançada da doença. A maioria dos casos registrados em mulheres resulta da transmissão sexual a partir do contato com homens bissexuais, usuários de drogas, promíscuos ou hemofílicos (22). A presença de sangue menstrual também aumenta significativamente o risco da transmissão do HIV (23). No presente estudo, o modo de transmissão do HIV foi semelhante para as mães brasileiras e norteamericanas, com a aquisição do vírus ocorrendo principalmente através de contato sexual com parceiro contaminado. Algumas vezes os pais ou responsáveis pela criança admitiam a hipótese de a mãe ter adquirido o HIV tanto por via sexual quanto pelo uso de drogas injetáveis. Poucos casos de transmissão do HIV foram atribuídos apenas ao uso de drogas injetáveis, por transfusão de sangue ou transmissão ocupacional. Quatro mães naturais brasileiras e uma mãe norteamericana eram HIV-negativas e seus filhos adquiriram o HIV por outros meios de transmissão que não a vertical. Mesmo assim, nossos resultados corroboram os dados epidemiológicos brasileiros e mundiais que apontam a transmissão vertical como principal via de transmissão do HIV em crianças (1, 9, 21).

Observamos que muitas das famílias chegaram ao diagnóstico da infecção por HIV através do adoecimento das crianças. Muitas das mães brasileiras afirmaram nunca ter realizado acompanhamento médico pré-natal. Outras afirmaram ter amamentado seus filhos mesmo sabendo dos riscos de transmissão do HIV por não terem acesso a alimentos que substituíssem o leite materno (24).

Existem muitas diferenças entre a AIDS em adultos e em crianças, incluindo a freqüência das manifestações estomatológicas (1, 15, 17). No presente estudo, cabe salientar que linfadenopatia cérvico-facial, aumento de volume das parótidas, candidíase eritematosa, petéquias, gengivite e xerostomia foram as manifestações mais freqüentes.

Muitas das famílias brasileiras apresentavam sérias dificuldades em administrar esquemas complexos de tratamento, fato este não observado nas famílias norte-americanas. Mesmo com regimes terapêuticos menos complexos, observamos que muitas famílias brasileiras apresentavam dificuldade em administrá-los, especialmente nos casos onde os avós eram os responsáveis pelas crianças. Em relação a isso, vale ressaltar o esforço das equipes médicas do

\section{REFERÊNCIAS}

1. Cotran RS, Kumar V, Robbins SL. Robbins: patologia estrutural e funcional. $5^{\mathrm{a}}$ ed. Rio de Janeiro: Guanabara Koogan; 1996. Pp. 194204.
2. Neves I, Morgado M. Immunological evaluation of human immunodeficiency virus infected individual by flow cytometry. Mem Inst Oswaldo Cruz 2000;95(3):393-400.
Hospital São Lucas e do HCPA em facilitar o entendimento dos esquemas terapêuticos necessários, mediante estratégias como tabelas com os horários dos medicamentos (para serem afixadas nas paredes ou na porta de geladeira) e desenhos ou figuras dos medicamentos (mostrando a forma dos comprimidos ou frascos). Também é importante destacar o fato de que a equipe de AIDS pediátrica do hospital norte-americano apresentava um número maior de profissionais por paciente do que as equipes dos hospitais brasileiros; além disso, maior quantidade de material educativo encontrava-se disponível, facilitando o esclarecimento de dúvidas. As consultas eram mais demoradas, com transmissão de muitas informações. Infelizmente, as condições econômicas no Brasil e a grande demanda de pacientes não possibilitam o mesmo tipo de relação numérica profissional/paciente observada nos Estados Unidos. O nível socioeconômico e cultural das famílias brasileiras em geral também não permitia que muitas informações fossem transmitidas numa mesma consulta. Acreditamos que todos esses fatores tenham influenciado a adesão das crianças brasileiras ao tratamento. As manifestações estomatológicas encontradas com mais freqüência nas crianças brasileiras em relação às norteamericanas certamente também refletiram esta realidade.

Agradecimentos. Agradecemos às crianças e às equipes de AIDS pediátrica dos hospitais participantes deste trabalho; ao programa de Doutorado em Estomatologia Clínica da Pontifícia Universidade Católica do Rio Grande do Sul; à Coordenação de Aperfeiçoamento de Pessoal de Nível Superior (CAPES) pelas bolsas PICDT e de estágio no exterior concedidas à Liliane Janete Grando; e à Pontifícia Universidade Católica do Rio Grande do Sul e Universidade do Estado de Nova Iorque pelo apoio financeiro.
3. Kaposi's sarcoma and Pneumocystis pneumonia among homosexual men - New York City and California. MMWR 1981;30(25): 305-308. 
4. Gottlieb MS, Schroff R, Schanker HM, Weisman JD, Fan PT, Wolf RA, et al. Pneumocystis cariniipneumonia and mucosal candidiasis in previously healthy homosexual men: evidence of a new acquired cellular immunodeficiency. N Engl J Med 1981;305(24): 1425-1431.

5. Possible transfusion-associated acquired immune deficiency syndrome (AIDS) California. MMWR 1982;31(48):652-654.

6. Unexplained immunodeficiency and opportunistic infection in infants - New York, New Jersey, California. MMWR 1982;31(49):665- 667.

7. Brasil, Ministério da Saúde, Secretaria de Políticas de Saúde, Coordenação Nacional de Doenças Sexualmente Transmissíveis e AIDS. Boletim Epidemiológico — AIDS 1998;ano XI(4). [Disponível em: http://www.aids.gov. br/final/dados/bol_aids1.htm].

8. Lima ALM, Kiffer CR, Uip D, Oliveira MS, Leite OM. HIV / AIDS: Perguntas e respostas. São Paulo: Atheneu; 1996.

9. Brasil, Ministério de Saúde, Secretaria de Assistência à Saúde, Programa Nacional de DST/AIDS. $1^{\circ}$ Congresso Nacional de Prevenção das DST/AIDS. Salvador: Ministério da Saúde; 1996.

10. Fontes M, Hillis J, Wasek G. Estimativas do número de crianças em situação de risco de ficarem órfãs e desalojadas em razão da AIDS no Brasil [documentos de trabalho]. Brasília: UNICEF; 1996

11. Melo JJ. Evolução da síndrome de imunodeficiência adquirida (AIDS-SIDA) na clínica odontológica: o papel do cirurgião- dentista. Rev Inst Cienc Saude 1997; número especial; 7-12.

12. New York State Department of Health, AIDS Institute. Criteria for the medical care of children and adolescents with HIV Infection. New York: New York State Department of Health; 1998.

13. Grando LJ. Perfil social, manifestações estomatológicas e detecção dos vírus CMV, EBV, HPV e HSV na cavidade bucal de crianças brasileiras e norte-americanas infectadas pelo HIV [tese de doutorado]. Porto Alegre: Universidade Federal do Rio Grande do Sul; 2000.

14. New York State Department of Health, AIDS Institute. Oral health care for adults, adolescents and children with HIV infection. New York: New York State Department of Health; 1998.

15. Leggott PJ. Oral manifestations of HIV infection in children. Oral Surg Oral Med Oral Pathol Oral Radiol Endod 1992;73(2):87-92.

16. Revised pediatric HIV classification system. Pediatr Dent 1996;18(2):104-105.

17. Guidelines for the use of antiretroviral agents in pediatric HIV infection. New York: Working Group on Antiretroviral Therapy and Medical Management of HIV-Infected Children; 2000.

18. Melnick SL, Nowjack-Raymer R, Kleinman D, Swango PA. A guide for epidemiological studies of oral manifestations of HIV infection. Geneva: WHO; 1993.

19. Ramos-Gomes FJ, Flaitz C, Catapano P, Murray P, Milnes AR, Dorenbaum A. Classification, diagnostic criteria, and treatment recommendations for orofacial manifestations in HIV-infected pediatric patients. J Clin Ped Dent 1999;23(2):85-95.

20. Ferguson FS, Berentsen B, Nachman S. Experiences of a pediatric dental program for HIV-positive children: oral manifestations and dental diseases observed in 58 children. Em: Greenspan JS, Greeenspan D. Oral manifestations of HIV infection. Chicago: Quintessence; 1995. Pp. 240-246.

21. Albano F, Spagnuolo MI, Berni Canani R, Guarino A. Adherence to antiretroviral therapy in HIV-infected children in Italy. AIDS Care 1999;11(6):711-714.

22. Rosa AIV, Chaves EM, Maturana MA, Larangeira MGF, Naud P. HIV na mulher. Em: Sprinz E, Finkelsztejn A. Rotinas em HIV e AIDS. Porto Alegre: Artes Médicas; 1999. Pp. 99-110.

23. Silva CLO, Galvão NAM. HIV na criança. Em: Sprinz E, Finkelsztejn A. Rotinas em HIV e AIDS. Porto Alegre: Artes Médicas; 1999. Pp. 91-98.

24. Brasil, Ministério da Saúde, Secretaria de Assistência à Saúde, Coordenação Geral do Programa Nacional de DST/AIDS, Unidade de Promoção à Saúde e Assistência. Aleitamento $X$ mulheres infectadas pelo HIV: recomendações. Vol 14. Brasília: Ministério da Saúde; 1995.

Manuscrito recebido em 8 de julho de 2002. Aceito em versão revisada em 21 de fevereiro de 2003.
ABSTRACT

\section{The association between oral manifestations and the socioeconomic and cultural characteristics of HIV-infected children in Brazil and in the United States of America}

Objectives. This study had two objectives: (1) to investigate the socioeconomic and cultural characteristics of HIV-infected children and their families in relation to the presence of oral manifestations of AIDS and (2) to identify the most frequent oral manifestations of AIDS in the sample of children studied.

Methods. The sample consisted of a total of 184 children-both boys and girlsfrom 0 to 13 years old. The children were receiving care at two hospitals in the city of Porto Alegre, Brazil (the Hospital de Clínicas de Porto Alegre and the Hospital São Lucas da Pontifícia Universidade Católica), and at Stony Brook University Hospital, which is in the state of New York, United States. Data were analyzed using the chisquare test, Student's $t$ test, and analysis of variance.

Results. Of the 184 patients, 117 of them $(63.59 \%)$ were receiving care at the Hospital de Clínicas de Porto Alegre, 26 (14.13\%) at the Hospital São Lucas, and 41 (22.28\%) at Stony Brook University Hospital. In the Brazilian sample (the two hospitals in Porto Alegre) $42.66 \%$ of the children were living with their natural parents (mostly with the mother), while in the United States $56.10 \%$ of the children were living with foster families. Concerning income, $39.86 \%$ of the Brazilian families had a monthly income ranging from US\$ 180 to US\$ 450, and 33.57\% had no income or did not provide information. With the United States sample, we were not allowed to ask about income, but all the families were classified by the social workers as having a monthly income below US\$ 1000 . HIV was acquired through vertical transmission by $97.20 \%$ of the Brazilian children and by $97.56 \%$ of the children in the United States sample. Oral manifestations were more frequent in the Brazilian children $(72.73 \%)$ than in the children in the United States sample $(53.66 \%)(P=0.01)$.

Conclusions. The frequency of oral manifestations in the samples in both Brazil and the United States was influenced by socioeconomic and cultural conditions, family structure and income, access to information concerning AIDS, and adherence to treatment. 